\title{
Nonequilibrium statistical operator in the generalized hydrodynamics of fluids
}

\author{
B.B. Markiv, I.P. Omelyan, M.V. Tokarchuk \\ Institute for Condensed Matter Physics of the National Academy of Sciences of Ukraine, \\ 1 Svientsitskii Str., 79011 Lviv, Ukraine
}

Received July 7, 2009, in final form September 1, 2009

\begin{abstract}
The investigations of the classical interacting particles systems far from equilibrium using the Zubarev nonequilibrium statistical operator method are presented. The problem of the relaxation of a nonequilibrium state of a system to the state of molecular hydrodynamics is considered. The nonequilibrium statistical operator and the appropriate transport equations which describe such a relaxation process are obtained. Explicit expressions for dynamic structure factor and momentum-momentum time correlation function are obtained by solving the set of equations for time correlation functions and some numerical results are presented.
\end{abstract}

Key words: nonequilibrium statistical operator, time-correlation function, transport coefficient, Liouville operator

PACS: $05.60 .-k, 05.20 . D d, 52.25 . F i, 71.45 .-d$

\section{Introduction}

The investigations for nonequilibrium processes in classical and quantum systems far from equilibrium, nonequilibrium states of which have their own relaxation times are urgent in modern theory of nonequilibrium processes. In the investigations of such kind, the nonequilibrium statistical operator (NSO) method by Zubarev with some modifications is successfully applied [1-4]. In particular in $[1,4]$ a new interpretation of the method of NSO is given, in which the operation of taking an invariant part in NSO is treated as the averaging of quasiequilibrium statistical operator on distribution of the past lifetime of a system

$$
\varrho(t)=\int_{-\infty}^{t} p_{q}\left(t-t^{\prime}\right) \varrho_{q}\left(t^{\prime}\right) \mathrm{d} t^{\prime},
$$

where $p_{q}(y)$ is the distribution of the past lifetime of a system. Since in the Zubarev NSO method the effect of a history of a system on its present state is taken into account to describe the real states in nonequilibrium systems such as kinetic, hydrodynamic etc., the gamma distribution

$$
p_{q}(y)=\frac{\varepsilon(\varepsilon y)^{k-1}}{\Gamma(k)} \mathrm{e}^{-\varepsilon y},
$$

should be used; $\Gamma(k)$ is gamma function. Gamma distribution is applied to the systems, whose evolution has several stages (the number of these stages coincides with the gamma distribution order). If $k=1$ we obtain an exponential distribution of the past lifetime $p_{q}(y)=\varepsilon \mathrm{e}^{-\varepsilon y}$, which corresponds to the Zubarev interpretation and is correct for long lifetimes.

On the other hand, an important achievement of the nonequilibrium theory of fluids are the results in the theory of molecular hydrodynamics, based on which the collective modes, timecorrelation functions, and generalized transport coefficients for the Lennard-Jones model of simple fluids were investigated [5-7]. Since a definite nonequilibrium statistical operator corresponds to the state of molecular hydrodynamics, the issue of a nonequilibrium state of a system of interacting 
particles relaxing to the state of molecular hydrodynamics presents definite interest. Choosing a distribution of the past lifetime of a system in the form of gamma distribution of the order $k=2$

$$
p_{q}(y)=\varepsilon^{2} y \mathrm{e}^{-\varepsilon y}
$$

we can consider the relaxation of a nonequilibrium state of the system to the equilibrium that passes in two stages. Thus, the consideration of the earlier one, the relaxation to the state of molecular hydrodynamics, is the aim of this paper.

\section{Nonequilibrium statistical operator of molecular hydrodynamics}

The molecular hydrodynamics of spatially homogeneous simple fluids is based on the transport equations for the means $\left\langle\tilde{a}_{\vec{k}}\right\rangle^{t}=\left\{\left\langle\hat{n}_{\vec{k}}\right\rangle^{t},\left\langle\hat{\vec{j}}_{\vec{k}}\right\rangle^{t},\left\langle\hat{h}_{\vec{k}}\right\rangle^{t}\right\}$,

$$
\hat{n}_{\vec{k}}=\sum_{j=1}^{N} \mathrm{e}^{-i \vec{k} \vec{r}_{j}}, \quad \hat{\vec{j}}_{\vec{k}}=\sum_{j=1}^{N} \vec{p}_{j} \mathrm{e}^{-i \vec{k} \vec{r}_{j}}, \quad \hat{h}_{\vec{k}}=\hat{E}_{\vec{k}}-\left\langle\hat{E}_{\vec{k}} \hat{n}_{-\vec{k}}\right\rangle_{0}\left\langle\hat{n}_{\vec{k}} \hat{n}_{-\vec{k}}\right\rangle_{0}^{-1} \hat{n}_{\vec{k}},
$$

where (3) are Fourier components of the number of particles, momentum density, and generalized enthalpy density, respectively, and

$$
\hat{E}_{\vec{k}}=\sum_{j=1}^{N}\left(\frac{p_{j}^{2}}{2 m}+\frac{1}{2} \sum_{l \neq j=1}^{N} \Phi\left(r_{l j}\right)\right) \mathrm{e}^{-i \vec{k} \vec{r}_{j}}
$$

are the Fourier components of the total energy density. Here, $\Phi\left(r_{l j}\right)$ is the pair interaction potential for particles of mass $\mathrm{m}$ whose total number is $\mathrm{N}$ and whose phase-space coordinates are $\left(\vec{p}_{j}, \vec{r}_{j}\right)$, where $\vec{p}_{j}$ and $\vec{r}_{j}$ are the respective momentum and spatial-coordinate radius vectors, $r_{l j}$ is the interparticle distance, and $\vec{k}$ is the wave-vector. Such transport equations have the structure [8-10]

$$
\frac{\partial}{\partial t}\left\langle\hat{a}_{l, \vec{k}}\right\rangle^{t}-\sum_{m} i \Omega_{l m}(\vec{k})\left\langle\hat{a}_{m, \vec{k}}\right\rangle^{t}+\sum_{m} \int_{-\infty}^{t} \mathrm{e}^{\varepsilon\left(t^{\prime}-t\right)} \varphi_{l m}\left(\vec{k}, t, t^{\prime}\right)\left\langle\hat{a}_{m, \vec{k}}\right\rangle^{t^{\prime}} \mathrm{d} t^{\prime}=0,
$$

where we introduce the notation $l=1,2,3, \hat{a}_{1, \vec{k}}=\hat{n}_{\vec{k}}, \hat{a}_{2, \vec{k}}=\hat{\vec{j}}_{\vec{k}}, \hat{a}_{3, \vec{k}}=\hat{h}_{\vec{k}}$. In this equation $i \Omega_{m l}(\vec{k})=\sum_{l^{\prime}}\left\langle\dot{\hat{a}}_{m, \vec{k}} \hat{a}_{l^{\prime},-\vec{k}}\right\rangle_{0} \tilde{\Phi}_{l^{\prime} l}^{-1}(\vec{k})$ are the elements of the matrix

$$
i \tilde{\Omega}(\vec{k})=\left(\begin{array}{ccc}
0 & i \Omega_{n j} & 0 \\
i \Omega_{j n} & 0 & i \Omega_{j h} \\
0 & i \Omega_{h j} & 0
\end{array}\right)_{(\vec{k})}
$$

which describe nondissipative processes and are the normalized equilibrium correlation functions.

Furthermore, $\tilde{\Phi}_{l l^{\prime}}^{-1}(\vec{k})$ are the elements of the inverse of the matrix $\tilde{\Phi}(\vec{k})$ :

$$
\tilde{\Phi}(\vec{k})=\left(\begin{array}{ccc}
\left\langle\hat{n}_{\vec{k}} \hat{n}_{-\vec{k}}\right\rangle_{0} & 0 & 0 \\
0 & \left\langle\hat{\vec{j}}_{\vec{k}} \cdot \hat{\vec{j}}_{-\vec{k}}\right\rangle_{0} & 0 \\
0 & 0 & \left\langle\hat{h}_{\vec{k}} \hat{h}_{-\vec{k}}\right\rangle_{0}
\end{array}\right),
$$

whose structure indicates the orthogonality of the variables $\hat{n}_{\vec{k}}, \hat{\vec{j}}_{\vec{k}}$, and $\hat{h}_{\vec{k}}$ in the sense that $\left\langle\hat{n}_{\vec{k}} \hat{\vec{j}}_{-\vec{k}}\right\rangle_{0}=0,\left\langle\hat{n}_{\vec{k}} \hat{h}_{-\vec{k}}\right\rangle_{0}=0$, and $\left\langle\hat{\vec{j}}_{\vec{k}} \hat{h}_{-\vec{k}}\right\rangle_{0}=0$. Here, $\left\langle\hat{n}_{\vec{k}} \hat{n}_{-\vec{k}}\right\rangle_{0}=S_{2}(\vec{k})$ is the structure factor for the simple-fluid atoms, where the average $\langle\ldots\rangle_{0}=\int \mathrm{d} \Gamma_{N} \ldots \varrho_{0}\left(x^{N}\right)$ is taken using the static equilibrium operators $\varrho_{0}\left(x^{N}\right)=Z^{-1} \exp (-\beta(H-\mu N)), Z=\int \mathrm{d} \Gamma_{N} \exp (-\beta(H-\mu N))$ is the grand 
partition function, $\beta=1 / k_{\mathrm{B}} T, k_{\mathrm{B}}$ is the Boltzmann constant, $T$ is the equilibrium temperature value, $\mu$ is the chemical potential, and

$$
H=\sum_{j=1}^{N}\left(\frac{p_{j}^{2}}{2 m}+\frac{1}{2} \sum_{l \neq j=1}^{N} \Phi\left(r_{l j}\right)\right)
$$

is the Hamiltonian for the simple-fluid atoms.

The dissipative processes in molecular hydrodynamics equations (4) are described by the transport kernels

$$
\varphi_{l m}\left(\vec{k} ; t, t^{\prime}\right)=\sum_{l^{\prime}}\left\langle I_{l}(\vec{k}) T_{0}^{\mathrm{H}}\left(t, t^{\prime}\right) I_{l^{\prime}}(-\vec{k})\right\rangle_{0} \tilde{\Phi}_{l^{\prime} m}^{-1}(\vec{k}),
$$

which determine the generalized viscosity and heat-conduction coefficients and the transport crosscoefficient, where $I_{l}(\vec{k})=\left(1-P_{\mathrm{H}}\right) i L_{N} \hat{a}_{l, \vec{k}}$ are the generalized flows,

$$
i L_{N}=\sum_{j=1}^{N} \frac{\vec{p}_{j}}{m} \frac{\partial}{\partial \vec{r}_{j}}-\frac{1}{2} \sum_{l \neq j=1}^{N} \frac{\partial}{\partial \vec{r}_{j}} \Phi\left(r_{l j}\right)\left(\frac{\partial}{\partial \vec{p}_{j}}-\frac{\partial}{\partial \vec{p}_{l}}\right),
$$

is the Liouville operator corresponding to the Hamiltonian $H$ of the system, $P_{\mathrm{H}}$ is the Mori projection operator constructed on the hydrodynamic orthogonal variables $\hat{n}_{\vec{k}}, \hat{\vec{j}}_{\vec{k}}$, and $\hat{h}_{\vec{k}}$, and $T_{0}^{\mathrm{H}}\left(t, t^{\prime}\right)=\exp \left(\left(t^{\prime}-t\right)\left(1-P_{\mathrm{H}}\right) i L_{N}\right)$ is the evolution operator with regard to the Mori projection on the space of orthogonal dynamic variables $\hat{n}_{\vec{k}}, \hat{\vec{j}}_{\vec{k}}$, and $\hat{h}_{\vec{k}}$.

In the framework of the Zubarev nonequilibrium statistical operator method $[11,12]$, the molecular hydrodynamics equations (4) are derived using the nonequilibrium statistical operator [9]

$$
\begin{aligned}
\varrho^{\mathrm{H}}\left(x^{N} ; t\right)= & \left(1+\sum_{l, m} \sum_{\vec{k}}\left(\hat{a}_{l,-\vec{k}} \tilde{\Phi}_{l m}^{-1}(\vec{k})\left\langle\hat{a}_{m, \vec{k}}\right\rangle^{t}\right.\right. \\
& \left.\left.-\int_{-\infty}^{t} \mathrm{e}^{\varepsilon\left(t^{\prime}-t\right)} T_{0}^{\mathrm{H}}\left(t, t^{\prime}\right) I_{l}(-\vec{k}) \tilde{\Phi}_{l m}^{-1}(\vec{k})\left\langle\hat{a}_{m, \vec{k}}\right\rangle^{t^{\prime}} \mathrm{d} t^{\prime}\right)\right) \varrho_{0}\left(x^{N}\right),
\end{aligned}
$$

i. e., nonequilibrium statistical operator (10) corresponds to the state of molecular hydrodynamics of a simple fluid. The operator (10) is a function of the extended set of dynamical variables $\hat{n}_{\vec{k}}, \hat{\vec{j}}_{\vec{k}}$, and $\hat{h}_{\vec{k}}$ and generalized flows $I_{n}(\vec{k})=0, I_{j}(\vec{k})$, and $I_{h}(\vec{k})$, which determine generalized transport coefficients by means of (8).

\section{Relaxation to the state of molecular hydrodynamics}

Based on the results described above, we can now consider the problem of relaxation of the nonequilibrium state of the system to the state of molecular hydrodynamics. And since further we will use the Markovian approximation for transport kernels, the nonequilibruim statistical operator of molecular hydrodynamics (10) can be written in the form

$$
\begin{aligned}
\varrho^{\mathrm{H}}\left(x^{N} ; t\right) & =\varrho_{0}\left(x^{N}\right)\left\{1+\sum_{\vec{k}} \sum_{l m}\left\langle\hat{a}_{l, \vec{k}}\right\rangle^{t} \Phi_{l m}^{-1}(\vec{k})\left(\hat{a}_{m,-\vec{k}}-\hat{I}_{m}^{\mathrm{H}}(-\vec{k})\right)\right\}, \\
\hat{I}_{m}^{\mathrm{H}}(\vec{k}) & =\int_{0}^{\infty} \mathrm{e}^{-\varepsilon t^{\prime}} T_{0}^{\mathrm{H}}\left(t^{\prime}\right) \hat{I}_{m}(\vec{k}) \mathrm{d} t^{\prime} .
\end{aligned}
$$

Following the usual scheme [12] we will find the desired NSO from the modified Liouville equation with an infinitesimal source, which breaks the time-reversal symmetry and selects the retarded solutions of equation

$$
\left(\frac{\partial}{\partial t}+i L_{N}\right) \varrho\left(x^{N} ; t\right)=-\tilde{\varepsilon}\left(\varrho\left(x^{N} ; t\right)-\varrho^{\mathrm{H}}\left(x^{N} ; t\right)\right),
$$


but now $\tilde{\varepsilon}$ does not go to zero and, according to [4], can be treated as an inverse average lifetime of a system.

Presenting the statistical operator as a sum $\varrho(t)=\varrho^{\mathrm{H}}(t)+\Delta \varrho(t)$ and substituting it into $(13)$ results in equation for $\Delta \varrho(t)$ :

$$
\left(\frac{\partial}{\partial t}+i L_{N}+\tilde{\varepsilon}\right) \Delta \varrho(t)=-\left(\frac{\partial}{\partial t}+i L_{N}\right) \varrho^{\mathrm{H}}(t) .
$$

To solve (14) we have to exclude the derivative $\partial \varrho^{\mathrm{H}}(t) / \partial t$ from the equation, and this can be done even in the case of explicit dependence of $\varrho^{\mathrm{H}}(t)$ on time [13]

$$
\frac{\partial \varrho^{\mathrm{H}}(t)}{\partial t}=-P_{\mathrm{R}}(t) i L_{N} \varrho(t)+\left(\frac{\partial \varrho^{\mathrm{H}}(t)}{\partial t}\right)_{\operatorname{expl}},
$$

where

$$
P_{\mathrm{R}}(t) \varrho^{\prime}(t)=\sum_{l} \frac{\partial \varrho^{\mathrm{H}}(t)}{\partial\left\langle\hat{a}_{l, \vec{k}}\right\rangle^{t}} \operatorname{Tr}\left\{\hat{a}_{l, \vec{k}} \varrho^{\prime}(t)\right\}
$$

is the Robertson projection operator. Neglecting infinitesimal terms one obtains the simple representation for the explicit time derivative of $\varrho^{\mathrm{H}}(t)$

$$
\left(\frac{\partial \varrho^{\mathrm{H}}(t)}{\partial t}\right)_{\mathrm{expl}}=-\left(1-P_{\mathrm{H}}\right) i L_{N} \varrho^{\mathrm{H}}(t) .
$$

Since the $P_{\mathrm{R}}(t)$ acts on distribution functions, it is more convenient to pass to another projection operator which acts on dynamic variables. Thus, an action of the Robertson projection operator on $i L_{N} \varrho^{\mathrm{H}}(t)$ can be represented in the form

$$
P_{\mathrm{R}}(t) i L_{N} \varrho^{\mathrm{H}}(t)=\bar{P}_{\mathrm{H}} i L_{N} \varrho^{\mathrm{H}}(t),
$$

where $\bar{P}_{\mathrm{H}}$ is a new "projection" operator, which consists of two parts

$$
\begin{aligned}
\bar{P}_{\mathrm{H}} \ldots & =\sum_{l m}\left\langle\ldots \hat{a}_{l, \vec{k}}\right\rangle_{0} \Phi_{l m}^{-1}(\vec{k})\left(\hat{a}_{m,-\vec{k}}-\hat{I}_{m}^{\mathrm{H}}(-\vec{k})\right) \\
\bar{P}_{\mathrm{H}} & =P_{\mathrm{H}}-P^{\prime}
\end{aligned}
$$

and the time-dependent part has the following structure

$$
P^{\prime} \ldots=\sum_{l m}\left\langle\ldots \hat{a}_{l, \vec{k}}\right\rangle_{0} \Phi_{l m}^{-1} \hat{I}_{m}^{\mathrm{H}}(-\vec{k})
$$

Now combining (14)-(19) we can obtain the final form of the equation for $\Delta \varrho(t)$

$$
\left(\frac{\partial}{\partial t}+\left[1-P_{\mathrm{R}}(t)\right] i L_{N}+\tilde{\varepsilon}\right) \Delta \varrho(t)=-P^{\prime} i L_{N} \varrho^{\mathrm{H}}(t) .
$$

Its formal solution can be presented as

$$
\Delta \varrho(t)=-\int_{-\infty}^{t} \mathrm{e}^{\tilde{\varepsilon}\left(t^{\prime}-t\right)} T_{\mathrm{R}}\left(t, t^{\prime}\right) P^{\prime} i L_{N} \varrho^{\mathrm{H}}\left(t^{\prime}\right) \mathrm{d} t^{\prime},
$$

where the evolution operator with regard to projection $P_{\mathrm{R}}(t)$ is specified by the relation

$$
T_{\mathrm{R}}\left(t, t^{\prime}\right)=\exp _{+}\left(-\int_{t^{\prime}}^{t} \mathrm{~d} \tau\left[1-P_{\mathrm{R}}(\tau)\right] i L_{N}\right)
$$


Thus we obtain the NSO as a functional of means of dynamic variables, and we can use it to construct transport equations describing relaxation of the system to the state of molecular hydrodynamics. For this purpose let us use the relation

$$
\frac{\mathrm{d}\left\langle\hat{a}_{m, \vec{k}}\right\rangle^{t}}{\mathrm{~d} t}=\operatorname{Tr}\left\{\frac{\mathrm{d} \hat{a}_{m, \vec{k}}}{\mathrm{~d} t} \varrho(t)\right\}
$$

or

$$
\frac{\mathrm{d}\left\langle\hat{a}_{m, \vec{k}}\right\rangle^{t}}{\mathrm{~d} t}=\left\langle\frac{\mathrm{d} \hat{a}_{m, \vec{k}}}{\mathrm{~d} t}\right\rangle_{\mathrm{H}}^{t}+\operatorname{Tr}\left\{\hat{I}_{m}(\vec{k}) \Delta \varrho(t)\right\} .
$$

Using (11) and (20) we obtain the transport equations in general form

$$
\begin{aligned}
\frac{\partial}{\partial t}\left\langle\hat{a}_{l, \vec{k}}\right\rangle^{t}= & \sum_{m}\left(i \Omega_{l m}(\vec{k})-\varphi_{l m}(\vec{k})\right)\left\langle\hat{a}_{m, \vec{k}}\right\rangle^{t} \\
& +\sum_{m, n} \int_{-\infty}^{t} \mathrm{e}^{\tilde{\varepsilon}\left(t^{\prime}-t\right)} \varphi_{l m}^{R}\left(\vec{k} ; t, t^{\prime}\right)\left(i \Omega_{m n}(-\vec{k})-\varphi_{m n}(-\vec{k})\right)\left\langle\hat{a}_{n, \vec{k}}\right\rangle^{t^{\prime}} \mathrm{d} t^{\prime}
\end{aligned}
$$

with new memory functions

$$
\varphi_{l n}^{R}\left(\vec{k} ; t, t^{\prime}\right)=\sum_{m}\left\langle\hat{I}_{l}(\vec{k}) T_{\mathrm{R}}\left(t, t^{\prime}\right) \hat{I}_{m}^{\mathrm{H}}(-\vec{k})\right\rangle_{0} \Phi_{m n}^{-1}(\vec{k})
$$

The first term in the right-hand side describes the contribution of molecular hydrodynamics, and collective excitations are thoroughly studied to this end. Memory functions (26) have a complicated structure. It is interesting that the evolution of the reduced description parameters besides others is determined by the contributions of molecular hydrodynamics both in the present moment of time and in the previous moments of a history of the system.

Based on the transport equations (25) we can write down the set of equations for time correlation functions, which in Laplace representation have a form

$$
\begin{gathered}
i z \Phi(z, \vec{k})+\left(i \Omega_{l m}(\vec{k})-\varphi_{l m}(\vec{k})\right) \Phi(z, \vec{k})+\varphi^{\mathrm{R}}(z, \vec{k})\left(i \Omega_{l m}(-\vec{k})-\varphi_{l m}(-\vec{k})\right) \Phi(z, \vec{k})=-\Phi(\vec{k}), \\
z=\omega+i \tilde{\varepsilon}
\end{gathered}
$$

Since direct calculations of the transport kernels (26) are difficult we use the simple approximation

$$
\varphi_{l m}^{\mathrm{R}}(z, \vec{k}) \simeq \frac{\varphi_{l m}(\vec{k})}{i z}=\frac{\varphi_{l m}(\vec{k})}{i \omega-\tilde{\varepsilon}}
$$

( $\tilde{\varepsilon}=\tau^{-1}$ is inverse lifetime of a system in the nonequilibrium state that relaxes and passes to the state of molecular hydrodynamics). To simplify our calculations we will work in the visco-elastic approximation, when the only variables are the means of number of particles and momentum densities.

Thus solving the set of equations (27) one can calculate the time correlation functions of interest. For instance, the dynamic structure factor is given by

$$
S(k, \omega)=\frac{2 \varphi_{j j}(k) \Omega^{2}\left\{1-\left(\tau \varphi_{j j}(k)\right)^{2}\right\}}{\left\{\left(\omega^{2}-\Omega^{2}\right)\left(1-\tau \varphi_{j j}(k)\right)\right\}^{2}+\left\{\omega\left[\left(\omega^{2}-\Omega^{2}\right) \tau+\varphi_{j j}(k)\left(1-\tau \varphi_{j j}(k)\right)\right]\right\}^{2}},
$$

where

$$
\Omega^{2}=\frac{k^{2}}{m \beta S(k)} .
$$

For momentum-momentum transverse correlation function we obtained

$$
\Phi_{j j}^{T}(k, \omega)=\frac{\varphi_{j j}(k)\left(1+\tau \varphi_{j j}(k)\right)+\omega^{2} \tau^{2} \varphi_{j j}(k)}{\left\{\omega^{2} \tau+\varphi_{j j}(k)\left(1+\tau \varphi_{j j}(k)\right)\right\}^{2}+\left\{\omega\left(1-\tau \varphi_{j j}(k)\right)\right\}^{2}} .
$$



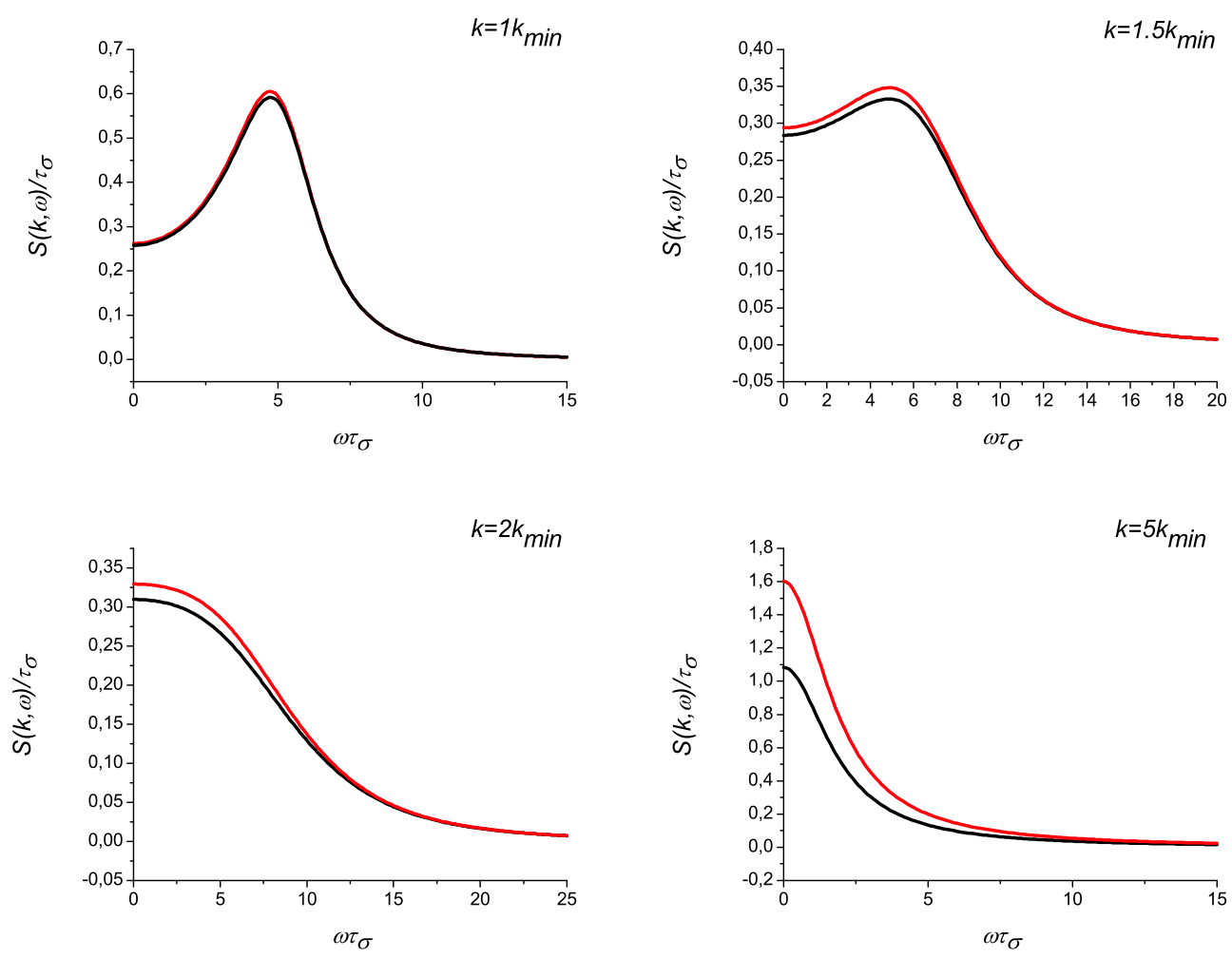

Figure 1. Dynamic structure factor of a LJ fluid as a function of frequency. The red curve is obtained using (29), and the black one represents molecular hydrodynamic calculations $[8,9]$.
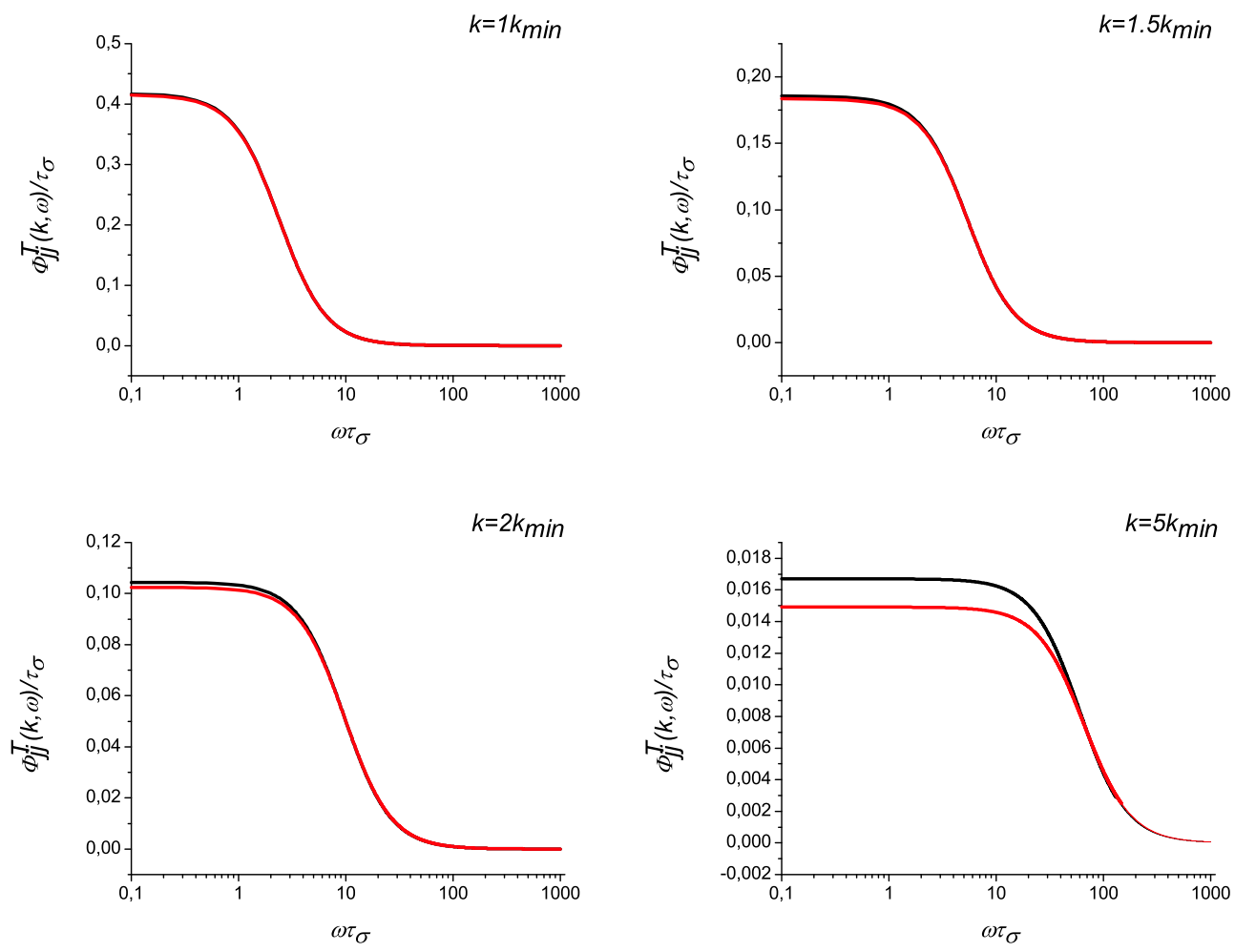

Figure 2. Momentum-momentum transverse correlation function of a LJ fluid as a function of frequency. The red curve is obtained using (30), and the black one is as in figure 1. 
Frequency dependence of these functions is shown in figure 1 and figure 2. The central peak of the dynamic structure factor is not present due to the absence of the heat mode in such a two variable formalism. All the numerical data are taken from [6]. Time correlation functions are calculated at reduced density $n^{*}=0.845$ and temperature $T^{*}=1.706$ for four values of wave-vector $k$, namely $k \sigma_{\mathrm{LJ}}=0.936=k_{\min } \sigma_{\mathrm{LJ}}, 1.404,1.872$, and 4.678. $\tau_{\sigma}=\left(\sigma_{\mathrm{LJ}}^{2} m / \epsilon_{\mathrm{LJ}}\right)^{1 / 2}$ is constructed of parameters of the problem for argon. The behaviors of these functions are generally similar to those of linear hydrodynamics $[8,9]$ and the difference is only in numerical data. Analyzing the behavior of time correlation functions one can determine that the time of such a kind of relaxation process is very short $\left(\sim 10^{-14} \mathrm{sec}\right)$. With the increase of $k$ we observe some kind of renormalization and the theory does not work at large $k$. It is natural that turning $\tau$ (i. e. the lifetime of the state under consideration) to zero we will reproduce the results of molecular hydrodynamics.

\section{Conclusions}

The characteristics of the state of molecular hydrodynamics for fluids (such as collective excitations, time-correlation functions, and transport coefficients) have now been widely studied, which can serve as a basis for considerations beyond the framework of molecular hydrodynamics. Considering the relaxation of a nonequilibrium state of the system to the state of molecular hydrodynamics with transport kernels in the Markovian approximation, which is thoroughly studied for the Lennard-Jones simple fluid, we tried to realize this approach concisely using the Zubarev nonequilibrium statistical operator method. It is determined that time of such kind of relaxation is very short $\left(\sim 10^{-14} \mathrm{sec}\right)$. Since the problem is considered in the Markovian approximation for transport kernels, the presented theory is not valid for large values of wave vector. The increase of $k$ results in nonphysical behavior of dynamic structure factor while decreasing it and turning $\tau$ to zero we obtain the well known results of linear hydrodynamics.

Evidently, the results depend on an approximation to be done for transport kernel $\varphi_{l m}^{\mathrm{R}}\left(\vec{k} ; t, t^{\prime}\right)$. The assumption (28) we used is proper on the short times of the system evolution (large frequencies). It is very interesting to investigate such a relaxation process when transport kernel can be approximated by Gaussian dependence on time

$$
\varphi_{j j}^{\mathrm{R}}(\vec{k} ; t) \sim \varphi_{j j}(\vec{k}) \mathrm{e}^{-\alpha\left(\varphi_{j j}(\vec{k}), \varphi_{\pi \pi}(\vec{k})\right) t^{2}}
$$

where $\alpha\left(\varphi_{j j}(\vec{k}), \varphi_{\pi \pi}(\vec{k})\right)$ is some functional of $\varphi_{\pi \pi}(\vec{k})=\left\langle i L_{N} \hat{I}_{j}(\vec{k}) i L_{N} \hat{I}_{j}(-\vec{k})\right\rangle_{0} \Phi_{j j}^{-1}(\vec{k})$, and $\varphi_{j j}(\vec{k})$ of molecular hydrodynamics. These investigations will be done in due time.

\section{References}

1. Ryazanov V.V., Fortschr. Phys., 2001, 49, 885.

2. Luzzi R., Vasconcellos A.R., Fortschr. Phys., 1990, 38, 887.

3. Ramos J.G., Vasconcellos A.R., Luzzi R., Fortschr. Phys., 1999, 47, 937.

4. Ryazanov V.V. Preprint arXiv: 0803.4111v1, 2008.

5. de Schepper I.M., Cohen E.G.D., Bruin C., van Rijs J.C., Montrooij W., de Graaf L.A., Phys. Rev. A, 1988, 38, 271.

6. Omelyan I.P., Mryglod I.M., Condens. Matter Phys., 1994, 4, 128.

7. Mryglod I.M., Condens. Matter Phys., 1998, 1, 753.

8. Boon J., Yip S. Molecular Hydrodynamics. McGraw-Hill Inc., New York, 1980, p. 237.

9. Mryglod I.M., Tokarchuk M.V., Problems of atomic science and technique. Series: Nuclear physics investigations (theory and experiment), 1992, 3(24), 134.

10. Markiv B.B., Omelyan I.P., Tokarchuk M.V., Theor. Math. Phys.,2008, 154, 75.

11. Zubarev D.N. Itogi Nauki i Tekhniki, Sovr. Prob. Mat., 1980, 15, 131.

12. Zubarev D.N., Morozov V.G., Repke G. Statistical Mechanics of Nonequilibrium Processes, vol. 1. Akademie, Berlin, 1996, p. 103.

13. Morozov V.G., Theor. Math. Phys., 2008, 154, 84. 


\title{
Нерівноважний статистичний оператор в узагальненій гідродинаміці рідин
}

\author{
Б.Б. Марків, І.П. Омелян, М.В. Токарчук \\ Інститут фізики конденсованих систем НАН України, вул. Свєнціцького, 1, 79011 Львів, Україна \\ Отримано 7 липня 2009 р., в остаточному вигляді - 1 вересня 2009 р.
}

\begin{abstract}
На основі методу нерівноважного статистичного оператора Д.Зубарєва проведено дослідження систем класичних взаємодіючих частинок далеко від рівноваги. Розглядається задача релаксації нерівноважного стану системи до стану молекулярної гідродинаміки. Отримано нерівноважний статистичний оператор та відповідні рівняння переносу, які описують такий релаксаційний процес. Розв'язуючи систему рівнянь для часових кореляційних функцій, отримано явні вирази для динамічного структурного фактора та часової кореляційної функції “імпульс-імпульс", а також приведено деякі числові результати.
\end{abstract}

Ключові слова: нерівноважний статистичний оператор, часові кореляційні функції, коефіцієнти переносу, оператор Ліувілля

PACS: $05.60 .-k, 05.20 . D d, 52.25 . F i, 71.45 .-d$ 